\title{
Period of Medical Insurance Coverage for Enrollment Basis
}

National Cancer Institute

\section{Source}

National Cancer Institute. Period of Medical Insurance Coverage for Enrollment Basis. NCI Thesaurus. Code C154528.

A time period of care where the start and stop dates are based on when the patient's costs are covered by their personal medical insurance plan. 